Western University

Scholarship@Western

FIMS Presentations

Information \& Media Studies (FIMS) Faculty

2005

\title{
Information Provision for Informed Prenatal Decision Making
}

Jacquelyn Burkell

The University of Western Ontario, jburkell@uwo.ca

Pamela J. McKenzie

University of Western Ontario, pmckenzi@uwo.ca

Follow this and additional works at: https://ir.lib.uwo.ca/fimspres

Part of the Communication Commons, and the Library and Information Science Commons

Citation of this paper:

Burkell, Jacquelyn and McKenzie, Pamela J., "Information Provision for Informed Prenatal Decision Making" (2005). FIMS

Presentations. 27.

https://ir.lib.uwo.ca/fimspres/27 
Jacquelyn Burkell

Pamela J. McKenzie

Faculty of Information Studies, The University of Western Ontario, London, Ontario

\title{
Information Provision for Informed Prenatal Decision Making
}

\begin{abstract}
:
In decision making in maternity care, midwives provide information to support clientcentred decisions. Although midwives are expected to maintain neutrality, a variety of concerns limit nondirectiveness. The impact of these concerns is explored through qualitative analysis of midwife-client interactions. The results provide insight into what counts as "information" in these interactions.

Résumé : Lors de la prise de décision dans les soins de maternité, les sages-femmes offrent des informations pour soutenir les décisions de leurs patientes. Bien que l'on suppose que les sages-femmes doivent conserver une certaine neutralité, de nombreuses préoccupations limitent le manque de directives. L'impact de ces préoccupations est exploré à travers une analyse qualitative des interactions entre sages-femmes et patientes. Les résultats présentent un aperçu de ce qui est considéré comme « information » dans ces interactions.
\end{abstract}

\section{Introduction}

The midwifery profession is of particular interest to LIS researchers because the midwifery model of care is based on a relationship in which the midwife provides the pregnant woman with the information and support necessary for making informed decisions about her care (Rooks 1999). The clinical midwifery setting permits a study of the ways that "information" is negotiated and constituted through the actions of individuals within a broader social context, whether or not they are present in the local setting (McKenzie 2004, 2005 in press). This paper further explores this context by focusing on the constraints within which the midwife operates when providing a client with information for clinical decision-making.

The midwife's role is to provide accurate and up-to-date information relevant to the woman's choices, ensuring that she understands the potential risks and benefits of all options. This role is consistent with both the legal and moral requirement of informed consent (Spindel and Suarez 1995) and the Code of Ethics of the College of Midwives of Ontario College of Midwives of Ontario 1994a), and with the desires of many pregnant women (Seguin, Therrien, Champagne, and Larouche 1989, Green, Coupland, and Kitzinger 1990, Kennedy 2000, Pelkonen et al. 1998, Harvey et al. 2002). 
The role outlined for the midwife in decision making interactions is that of a neutral and supportive information source. It is implicitly assumed that the client's values and preferences will drive the decision making process, and that the midwife will provide information as required to aid the client in reaching an informed decision. This is an essentially nondirective approach (Weil, 2000). Nondirective approaches are endorsed in a variety of health care contexts (Bartels et al., 1997), although there is widespread recognition of the various factors that reduce the ability of health care practitioners to maintain nondirectiveness (Bartels et al., 1997; Williams et al, 2002). Some authors have even proposed that the principle of nondirectiveness be replaced by a different ethos, one that focuses on the complex psychosocial nature of the provider/ client interaction (Weil, 2003). The question then arises: Do midwives in fact use a nondirective approach in supporting client decisions? If not, what are the factors that influence midwife directiveness?

This paper reports on the initial content and discourse analysis of the ways that midwives provide decision-relevant information (Gwyn and Elwyn, 1999) and, specifically, the context of nondirectiveness within these encounters. Data come from transcripts of prenatal midwifery clinic visits. From these transcripts we have extracted instances of the midwife's delivery of information as women make decisions about clinical issues. In this paper we describe interactions concerning a single clinical issue: screening for the presence of the Group B streptococcus bacterium (GBS).

\section{Nondirectiveness}

Nondirectiveness has been cited as an important principle in client-centred decision making, particularly within the context of genetic counseling (Weil, 2000). As health care has become more client-centred, there is growing recognition that health care decisions should be informed by the values and preferences of the client paired with the clinical expertise of the health care professional. The nondirective approach suggests that patientcentred decisions can be achieved only if the health care professional ensures that each alternative is fully and equally described to the client, and if the health care provider maintains a strictly neutral stance, neither promoting nor endorsing any particular alternative.

Although in principle the nondirective approach is clearly desirable, in practice is has proven difficult if not impossible to achieve. Both qualitative (Williams et al., 2002) and quantitative (Bartels et al., 1997) studies of health care providers with respect to the issue have identified a variety of factors that reduce the ability of providers to maintain a nondirective approach. Providers recognized the inherent difficulty in being completely nondirective, acknowledging that subtle aspects of information presentation can influence client decisions; furthermore, they identified nondirectiveness as a clear departure from the more usual pattern of practitioner-patient interaction, in which the practitioner regularly offered opinions regarding the best course of action. When asked to identify specific reasons for departing from nondirectiveness, practitioners identified a variety of factors. First among these is direct response to clients: practitioners reported providing more direction when clients request their opinion, and they indicate that to refuse to 
provide this information would strain the provider/patient relationship. When clients are unable to reach a decision, providers step in to offer clarification or reflection, and these often take the form of more directive intervention. Sometimes providers move away from nondirectiveness in response to their own clinical expertise and knowledge. When clients seem disposed toward a decision that is suboptimal according to the best clinical evidence, providers feel a responsibility to promote an alternative course of action. Thus, in the context of genetic counseling, complete nondirectiveness is compromised in response to client needs and clinical knowledge. Other factors that might result in directiveness in consultation include personal opinions and values and institutional directiveness that indicates one course of action is preferable (Weil, 2003).

Recognition of the difficulties inherent in a strictly nondirective approach has led to reconsideration of the principle of nondirectiveness in genetic counseling. Instead of attempting (and largely failing) to maintain a strictly neutral position, genetic counselors are encouraged to recognize the full complex of issues represented in the decision making encounter, and to reflect on the factors that limit their own ability to neutrally support client decisions (Weil, 2003).

\section{Midwifery care in Ontario}

Midwifery is a licensed and publicly-funded profession in Ontario. Midwifery services are limited to women who are at low risk of complications, and midwives are mandated to consult and possibly transfer care to another health care professional should complications (including the need for induction of labour) arise in the course of a pregnancy and birth. For women under midwifery care, midwives provide primary prenatal care, attend home or hospital births, and provide six weeks of postpartum follow-up. The midwifery model in Ontario stresses the importance of informed choice with respect to decisions in maternity care. The College of Midwives of Ontario requires registered midwives to "provide each client with the following information throughout the course of care: potential benefits and risks of, and alternatives to, procedures, tests, and medications; relevant research evidence; community standards and practices" (College of Midwives of Ontario, 1994b).

The midwifery model in Ontario is based on "respect for pregnancy as a state of health and childbirth as a normal physiologic process" (College of Ontario Midwives, 1994a); thus, the philosophy stresses the non-medical nature of pregnancy and birth. At the same time, midwives are an integrated part of the health care system, with each midwife in Ontario maintaining privileges in at least one hospital. Midwives work collaboratively with other health care professionals, and support women in making choices about pregnancy and birth, including alternative choices of birthplace (hospital or home), and choices about issues such as pain medication during birth. This last issue illuminates the integrated nature of midwifery and other health care services. If a woman under midwifery care opts for pain medication, it must be provided by another health care professional, but the midwife continues to be the primary birth attendant unless complications develop. 
Midwives, therefore, practice within a complex network of social and professional responsibilities and associations (McKenzie 2005, in press). Midwives must balance the perspective that birth is a natural process with recognition of the value of medical interventions and approaches. At the same time, the midwife is committed to a fundamental respect for each woman and her individual needs and choices; midwives must also attend to relationships with hospitals and other health care professionals. Each of these relationships and responsibilities must influence the midwife in the practice of midwifery care, and each consideration influences the ability of the midwife to maintain a strictly neutral stance in interactions with clients (see Figure 1).

Figure 1: Concerns affecting nondirectiveness

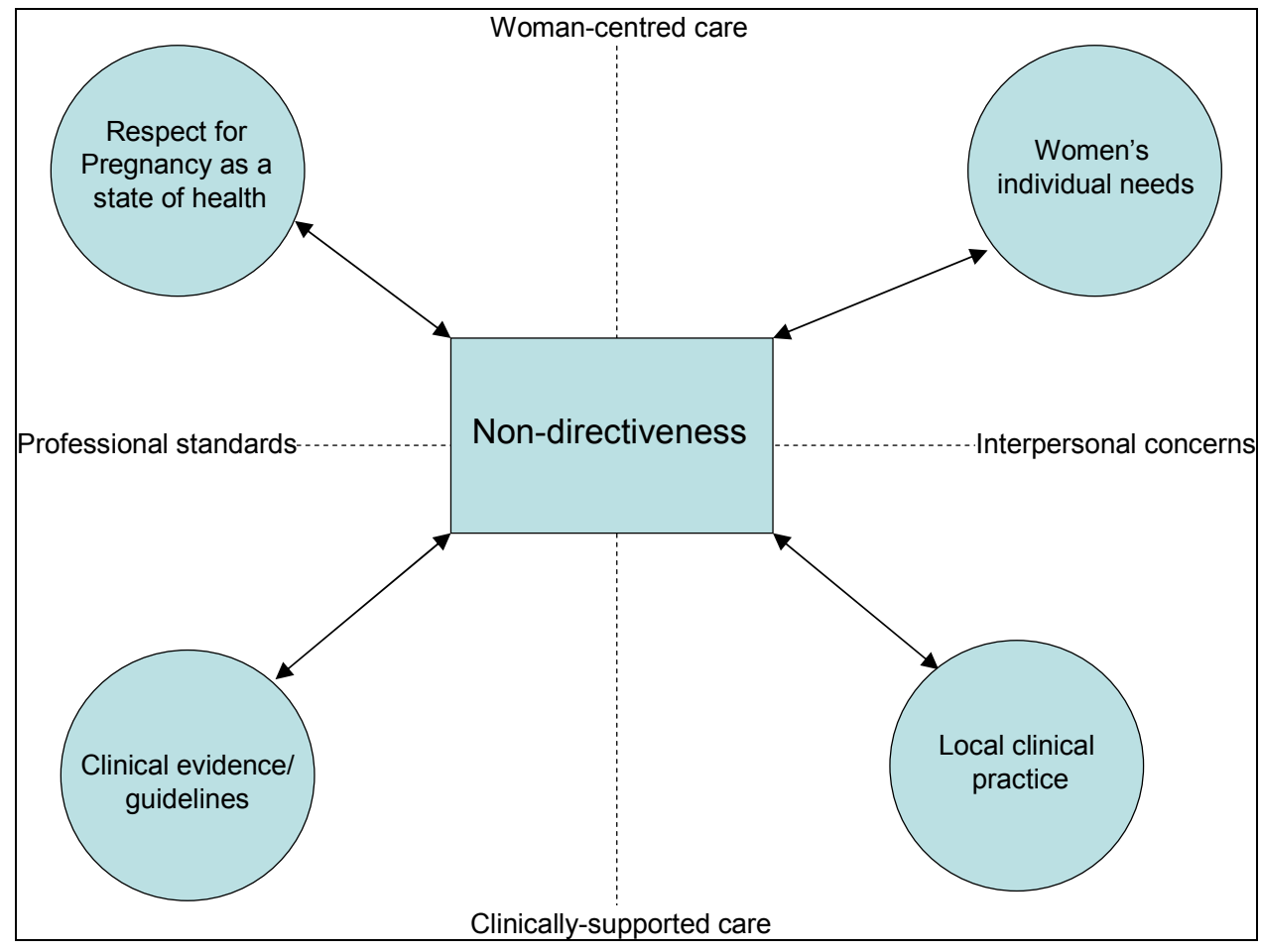

\section{The clinical context of Group B streptococcus screening}

One of the choices faced by pregnant women regards testing for Group B streptococcus (GBS), a commonly-occurring bacterium. GBS is part of normal intestinal and vaginal flora and is present in between $10 \%$ and $30 \%$ of women. Although GBS colonization is typically temporary and asymptomatic, the bacterium has been associated with a variety of adverse pregnancy outcomes, including preterm labour and postpartum maternal infection. The most significant concern is that GBS can be transferred to infants at birth, with potential serious negative effect. Neonatal GBS infection is the leading cause of life-threatening infectious disease in newborns, with mortality rates for GBS-infected 
neonates ranging from $5 \%$ to $20 \%$. The risk factors for neonatal infection include premature labour, fever in labour, prolonged rupture of membranes (18 hours or more before delivery), previous GBS-infected baby, and a GBS-related urinary tract infection during the current pregnancy. The overall incidence of neonatal GBS disease in Canada is 0.64 per thousand live births, with $57 \%$ of these cases occurring within the first week of a newborn's life ("early-onset") and the remainder developing between one week and several months after birth ("late-onset," Money and Dobson 2004).

Research demonstrates that the administration of intravenous antibiotics to GBS-positive women during labour significantly reduces the risk of newborn infection. There is, however, currently no available test that can accurately determine the GBS status of a woman when she is in labour. The culture method presently in use requires at least 36 hours for results, and the test therefore cannot be used to determine GBS status once labour has begun. Instead, the test is typically used for screening between 35 and 37 weeks gestation. Given the transient and variable nature of GBS colonization, the predictive value of these cultures for GBS status at time of delivery is not perfect (Ladhani, 2003); instead, screening at 35 to 37 weeks gestation is between 69 and 87 percent accurate in determining GBS status at time of delivery (Money and Dobson, 2004). Nonetheless, in the current clinical context the screening test provides the best possible information regarding the GBS status of a woman at the time of delivery.

Since the mid-1980s, a variety of strategies have been employed to reduce the risk of neonatal GBS disease. In 1996, the American Centers for Disease Control (CDC) recommended a two-pronged strategy for managing GBS in pregnancy. They endorsed screening for GBS, using a vaginal and rectal swab late in pregnancy (35-37 weeks gestation) to identify women who had the bacteria, suggesting that all women who tested positive should receive antibiotics in labour. In addition, they recommended a "riskfactor" approach, which advocated administering antibiotics to women in labour regardless of current GBS colonization status if they demonstrated any of the factors (identified above) that are associated with GBS infection. In June of 1997 the Society of Obstetricians and Gynaecologists of Canada (SOGC) adopted a similar strategy in which both universal screening at 35 to 37 weeks gestation followed by selective antibiotic administration and the administration of antibiotics to untested women with risk factors were acceptable options (Money and Dobson 2004).

Under the 1997 protocol, more than $80 \%$ of individual Ontario hospitals had policies with regard to GBS testing and intervention. In hospitals with a risk-based policy, $40-80 \%$ of deliveries at risk were getting antibiotics. In hospitals with screening policies, close to $90 \%$ of women delivering had been tested for GBS, and close to $90 \%$ of GBS-positive women were given antibiotics (Ladhani, 2003).

Further research has led to a widespread re-evaluation of GBS guidelines, first in the United States and later in Canada. The Centers for Disease Control revised its recommendations in 2002, advocating a universal screening approach. The American College of Obstetricians and Gynecologists followed suit. In Canada, the Canadian Task Force on Preventive Health Care (Shah and Ohlsson 2001) advocated universal screening 
followed by selective administration of antibiotics to GBS-positive women with other risk factors. In September of 2004 the Society of Obstetricians and Gynaecologists of Canada revised its clinical practice guidelines to advise universal late-pregnancy screening, and the treatment with intravenous antibiotic in labour of all women who tested GBS positive, as well as those with risk factors regardless of current GBS status for all women giving birth at term.

The issues for informed decision-making for pregnant women are obviously complex, contentious, and ever-changing. There are potentially three decision points for a pregnant woman: first, to screen or not to screen; second, to treat or not to treat if she tests positive for GBS but has no other risk factors; and, third, to treat or not to treat if she develops risk factors regardless of her GBS status. The first two of these decisions are made within the context of the midwife-client relationship. The third decision (if required) would most likely involve an obstetrician, since The College of Midwives of Ontario requires a consultation with an obstetrician for "prolonged rupture of membranes" (College of Midwives of Ontario 2000), which is the risk factor most commonly identified as a trigger for antibiotic administration. It should be noted, however, that the definition of "prolonged" varies from community to community; thus, community standards influence the length of time before antibiotic administration must be considered.

\section{Methodology}

This paper is based on an ongoing study of communication and information exchange in midwife-client visits in Ontario conducted by the second author. Data for this paper are taken from transcripts of audio-recordings of 36 prenatal midwife-client clinic visits from 15 southern Ontario midwifery practices. Nine visits took place in the city of Toronto (5 practices, with a community population of over 2 million and access to a number of tertiary-care teaching hospitals), 9 visits took place in large-city practices ( 2 practices, 300 000-900 000 people and access to one or more teaching hospitals), 11 visits took place in practices located in smaller cities (5 practices, 100 000-300 000 population and no teaching hospital) and 7 visits took places in practices located in small towns and villages ( 3 practices, population under 50000 , with a small local hospital or access to a hospital in a neighboring community only). Geographic regions were sampled purposively to maximize variation of community size and hospital access. Within each region, a convenience sample of willing practices was recruited, then willing midwives within each practice, and finally willing clients of those midwives. Some practices refused, and in some cases even though both practices and midwives agreed to participate there were no clients were willing to participate in the research.

The research has been funded by grants from The University of Western Ontario (both authors) and the Social Science and Humanities Research Council of Canada (second author), and data collection and analysis conform to both organizations' ethical guidelines on research on human subjects. 
All of the 36 visits were repeat visits to the midwifery clinic, although some were the first visit between the woman and the particular midwife (since clients typically interact with more than one midwife in the course of their care). Clients ranged from 14 to 40 weeks pregnant at the time of the recorded visit. Between two and five people were present for each visit. In addition to the midwife and the client, members of the pregnant woman's family or support network attended some visits (child(ren), partner, friend, family member). One or more midwifery students also participated in some prenatal visits as part of their clinical training.

Within the 36 transcripts, there are numerous instances of decision-making with respect to clinical issues. For this paper we have selectively analyzed the five interactions relating to Group B strep. The examples presented here were therefore chosen, not for their representativeness, but rather because they demonstrate directiveness in the interaction motivated by a variety of factors.

\section{Analysis}

Within the recorded interactions, information provision was generally comprehensive, with one notable exception: in no instance did the midwife indicate to the client that the results of the GBS screen are anything less than perfectly predictive of GBS status at the time of delivery. Instead, the screen is described in language such as this:

Yeah, and so, if you're, if you're a person who would say, "You know what? I don't want to be, I don't want to do anything [to induce labour] at 12 hours [after the rupture of membranes], " knowing that you're Group B Strep positive would help you make that decision.

The GBS swab is universally presented as a way of knowing GBS status at the time of delivery, and swab results are treated as definitive with respect to status at the time of delivery:

So we'll get this, we should get this back by, uh, next week and then if it was positive then we can just send you for the consult, with an ob? Just to get, an order for antibiotics on your chart?

It is worth considering why midwives might present the GBS swab in this way, with respect to the various pressures and responsibilities identified above.

The presentation of swab results as perfectly predictive of GBS status at the time of delivery (or omission of the information that results are less than perfectly predictive) is consistent with the position adopted by various governing bodies, including the Society of Obstetricians and Gynaecologists of Canada. Thus, in omitting this information the midwife is aligning her information giving practice with that adopted in the broader medical community based on the best available medical evidence. This responds to one imperative experienced by the midwife: the imperative to represent clinical evidence and guidelines in the decision making process. 
At the same time, introducing the uncertainty associated with GBS screening is likely to have various negative effects that impinge on a variety of additional considerations held by the midwife. First, it is likely that the additional uncertainty will cause stress for at least some women considering the screening test. On its own, this consideration for the psychological state of clients might not be enough to justify withholding of information, but the practice is supported by the earlier noted consistency with the information provided by other stakeholders within the medical community. This practice of protecting clients from potentially harmful effects of information is demonstrated in other circumstances where information is withheld to protect clients from overload or other negative results (Levy, 1999a, 1999b), and it responds to a second imperative experienced by midwives: the imperative to respond to the individual needs of clients.

Explicit discussion of the uncertainty of screening test results is likely to reduce screening test uptake in the context of GBS screening, consistent with choices regarding other types of screening tests (Wolf and Schorling 1998). Thus, midwives interested in maximizing screening test uptake would be less likely to focus on test uncertainty in their presentations to clients. Midwives have two intertwined interests that support the administration of the GBS swab, even if results are uncertain.

First, the principle of non-medicalized birth espoused in the midwifery philosophy means that, within the context of midwifery care, induction of labour through the use of drugs is less preferable to the natural onset of labour. With respect to this issue, GBS presents a particular challenge, since prolonged rupture of membranes has been shown to be a risk factor for GBS transfer to neonates, and thus reason both to induce labour and to treat with antibiotics in either of two cases: if a previous GBS swab has returned a positive result, or if no swab has been completed. Given the goal of avoiding induction and antibiotics, the best approach is to have the results of the GBS swab available.

Second, within many communities of practice the GBS swab is the community standard. Midwives practice in close association with local clinicians, but the integration of midwifery care into the health care system is a relatively recent development, and the relationship is at times challenging (Bourgeault, Benoit, and Davis-Floyd 2004). In particular, the scope of practice defined for midwives means that if complications develop the midwife must at the very least negotiate practice decisions with another health care provider, and in some cases is required to transfer care to another provider. In either event, compliance with local practice will protect the interests of both the midwife (from potential criticism should accepted practice not be followed) and the client (since the midwife will be in a better position to argue for the choices of her client with respect to issues such as induction if in all other respects the care adheres to accepted practice).

\section{Discussion}

The examples analyzed above demonstrate various instances in which midwives move away from a strictly nondirective stance in their interaction with clients. As we have argued, these departures are motivated by a variety of factors. Broadly conceptualized, 
midwifery requires a balance between woman-centred and clinically-supported care, and a balance between professional standards and interpersonal concerns. As a result, in decision making interactions the midwife simultaneously holds four different concerns: respect for pregnancy as a state of health, responsiveness to the (expressed or perceived) needs of the individual woman, clinical evidence and guidelines arising from the medical model, and local clinical practice and relationships (see Figure 1). Each of these concerns is legitimate within the decision making context, and responsiveness to any one of them can result in a departure from nondirectiveness.

Nondirectiveness must be considered in context. Midwives, as practitioners in genetic counseling, should recognize that nondirectiveness may be both impossible to achieve and inappropriate in many contexts. In fact, explicit guidance from midwives may be welcomed by women (Blix-Linström et al., 2004). As Suter (1998) argues, it may in fact be inappropriate, since health care professionals have expertise that is relevant to decision making:

They have witnessed clients of all sorts make difficulty choices: they have observed the kinds of factors that clients consider in making these decisions; and they have some knowledge of how clients respond to the decisions they make. (p 162-163)

While it is critical that the midwife's values and preferences not drive the decision process, there is clearly an appropriate place for the influence of the midwife's concerns on the decision making process. Holding in mind the higher level goal of ensuring the best possible birth experience for each individual woman, the midwife must act to balance the variety of interests she represents in the decision making process. Women who choose midwifery are at least in part drawn by the non-medical approach, and thus it is appropriate that the midwife, likely more experienced in this respect than her client, hold this principle in focus while providing decision support information. At the same time, clients are relying on the clinical expertise of midwives to ensure a safe birth and good outcome for both mother and child; at some level, therefore, it is critical that the midwife maintain a focus on clinical knowledge with respect to the decision making process to ensure that decisions are not inconsistent with acknowledged best practices. The extended association of midwife and client results is also an integral part of midwifery care; thus, it is important to clients that the midwife 'knows' them, and it is expected that the midwife will develop an understanding of the needs, values, and preferences of the client, and use this knowledge in practice, including in the context of decision making. Finally, the client relies on the good relationship between the midwife and other health care professionals to ensure choice of birthplace and appropriate medical intervention should complications arise. Midwives, therefore, must act to protect this interest in their interactions with clients.

In the GBS example, the four concerns we have identified collectively contribute to a particular form of nondirectiveness. With regard to other clinical tests and procedures, the tension and/or equilibrium among these concerns affects directiveness in different ways. The four concerns arise from midwives' relationship with the broader context within which they practice. A fuller analysis of the ways that midwives inform clients of their options will therefore provide insight into what counts as "information" in this 
setting, whose information counts, and how the interests of local and extralocal participants come to be represented and negotiated through the process of "informing" and "seeking information." 


\section{References}

Bartels, Dianne, M., Bonnie S. LeRoy, Patricia McCarthy, and Arthur L. Caplan. 1997. Nondirectiveness in genetic counselling: A survey of practitioners. American Journal of Medical Genetics, 72, 172-179.

Blix-Lindström, Sabine, Kyllike Christensson, and Eva Johansson. 2004. Women's satisfaction with decision-making related to augmentation of labour. Midwifery 20. 104-112.

Bourgeault, Ivy L., Cecilia Benoit, and Robbie Davis-Floyd, R., eds. 2004. Reconceiving Midwifery. Montreal: McGill-Queen's University Press

College of Midwives of Ontario. 1994a. Code of ethics. http://www.zfcconsulting.com/webprojects/midwives/mss/home/docs/CodeEthics.pdf

College of Midwives of Ontario. 1994b. Informed choice standard. http://www.zfcconsulting.com/webprojects/midwives/mss/home/docs/Informed\%20Choice.pdf

College of Midwives of Ontario. 2000. Indications for mandatory discussion, consultation, and transfer of care. 


\section{http://www.zfc-}

consulting.com/webprojects/midwives/mss/home/docs/Indications\%20for\%20Tra nsfer.pdf

Green, Josephine. M., Vanessa A. Coupland, and Jenny V. Kitzinger. 1990. Expectations, experiences, and psychological outcomes of childbirth: a prospective study of 825 women. Birth, 17(1), 15-24.

Gwyn, Richard and Glyn Elwyn. 1999. When is a shared decision not (quite) a shared decision? Negotiating preferences in a general practice encounter. Social science \& medicine 49(4): 437-47.

Harvey, Sheila; Rach, Diane; Stainton, M. Colleen; Jarrell, John; Brant, Rollin. 2002. Evaluation of satisfaction with midwifery care. Midwifery, 18(4), 260-267.

Kennedy, Holly Powell. 2000. A model of exemplary midwifery practice: results of a Delphi study. Journal of Midwifery \& Women's Health, 45(1), 4-19.

Levy, Valerie. 1999a. Midwives, informed choice, and power, Part 1. British Journal of Midwifery, 7(9), 583-6. 
Levy, Valerie. 1999b. Protective steering: a grounded theory study of the processes by which midwives facilitate informed choices during pregnancy. Journal of Advanced Nursing, 29(1), 104-112.

Linell, Per, and Margaretta Bredmar. 1996. Reconstructing Topical Sensitivity: Aspects of Face-Work in Talks between Midwives and Expectant Mothers. Research on Language and Social Interaction, 29, 347-379.

McKenzie, Pamela J. 2004. Positioning theory and the negotiation of information needs in a clinical midwifery setting. Journal of the American Society for Information Science and Technology 55(8): 685-694.

McKenzie, Pamela J. In press 2005. Mapping textually-mediated information practice in clinical midwifery care. In: Spink, Amanda, and Charles Cole, eds. New Horizons in Human Information Behavior. Springer.

Money, Deborah M. and Simon Dobson. 2004. The prevention of early-onset neonatal Group B Streptococcal disease. JOGC: Journal of Obstetrics and Gynaecology Canada 26(9): 826-32.

Pelkonen, Marjaana, Marja- Leena Perälä, and Katri Vehviläinen-Julkunen. 1998. Participation of expectant mothers in decision making in maternity care: results of a population-based survey. Journal of Advanced Nursing, 28(1), 21-29. 
Pilnick, Alison. 2004. 'It's just one of the best tests that we've got at the moment': the presentation of nuchal translucency screening for fetal abnormality in pregnancy. Discourse \& Society, 15(4), 451-65.

Pilnick, Alison. 2002. 'There are no rights or wrongs in these situations': identifying interactional difficulties in genetic counselling. Sociology of Health and Illness, 24(1), 66-88.

Rooks, Judith P. 1999. The midwifery model of care. Journal of Nurse-Midwifery, 44(4), $370-374$.

Salkovsis, Paul M., Dennis, Ruth, and Abigail L. Wroe. 1999. An experimental study of influences on the perceived likelihood of seeking genetic testing: "Nondirectiveness" may be misleading. Journal of Psychosomatic Research, $47(5), 439-447$.

Santalahti, Päivi, Elina Hemminki, Anne-Maria Latikka, and Markku Ryynänen. 1998. Women's decision-making in prenatal screening. Social Science \& Medicine 46(8), 1067-1076.

Seguin L.R., Therrien, F. Champagne, and D Larouche. 1989. The components of women's satisfaction with maternity care. Birth. 16(3):109-13. 
Shah, V. and A. Ohlsson, with the Canadian Task Force on Preventive Health Care. 2001. Prevention of early-onset Group B streptococcal (GBS) infection in the newborn: systematic review and recommendations. CTFPHC Technical Report \#01-6. London: Ontario: Canadian Task Force.

Spindel Peggy Garland and Suarez, Suzanne Hope. 1995. Informed consent and home birth. Journal of Nurse-Midwifery. 40(6):541-54.

Suter, Sonia. 1998. Value neutrality and nondirectiveness: Comments on "Future direction in genetic counselling". Kennedy Institute of Ethics Journal, 8.2, 161163.

Weil, Jon. 2000. Psychosocial Genetic Counseling. New York: Oxford University Press.

Weil, Jon. 2003. Psychosocial Genetic Counseling in the post-nondirective era: A point of view. Journal of Genetic Counseling, 12(3), 199-211.

Williams, Clare, Priscilla Alderson, and Bobbie Farsides. 2002. Is nondirectiveness possible within the context of antenatal screening and testing? Social Science and Medicine, 54, 339-347. 
Wolf, AM and JB Schorling. 1998. Preferences of elderly men for prostate-specific antigen screening and the impact of informed consent. Journal of Gerontololgy: A Biological Science and Medical Science, 53(3), 195-200. 